\title{
THIRD HANKEL DETERMINANT FOR CERTAIN SUBCLASS OF $p$-VALENT ANALYTIC FUNCTIONS
}

\author{
D. VAMSHEE KRISHNA \\ This paper is dedicated to Professor T. RAMREDDY on his $72^{\text {nd }}$ birthday
}

Received 15 October, 2015

Abstract. The objective of this paper is to obtain an upper bound to the third Hankel determinant for certain subclass of $p$-valent functions, using Toeplitz determinants.

2010 Mathematics Subject Classification: 30C45; 30C50

Keywords: p-valent analytic function, upper bound, Hankel determinant, positive real function, Toeplitz determinants

\section{INTRODUCTION}

Let $A_{p}$ denote the class of functions $f$ of the form

$$
f(z)=z^{p}+a_{p+1} z^{p+1}+\cdots,
$$

in the open unit disc $E=\{z:|z|<1\}$ with $p \in \mathbb{N}=\{1,2,3, \ldots\}$. Let $S$ be the subclass of $A_{1}=A$, consisting of univalent functions. In 1985, Louis de Branges de Bourcia proved the Bieberbach conjecture, i.e., for a univalent function its $n^{\text {th }}$ - coefficient is bounded by $n$ (see [3]). The bounds for the coefficients of these functions give information about their geometric properties. In particular, the growth and distortion properties of a normalized univalent function are determined by the bound of its second coefficient. The Hankel determinant of $f$ for $q \geq 1$ and $n \geq 1$ (when $p=1$ ) was defined by Pommerenke [10] as follows and has been extensively studied.

$$
H_{q}(n)=\left|\begin{array}{cccc}
a_{n} & a_{n+1} & \cdots & a_{n+q-1} \\
a_{n+1} & a_{n+2} & \cdots & a_{n+q} \\
\vdots & \vdots & \vdots & \vdots \\
a_{n+q-1} & a_{n+q} & \cdots & a_{n+2 q-2}
\end{array}\right| .
$$

One can easily observe that the Fekete-Szegô functional is $H_{2}(1)=a_{3}-a_{2}^{2}$. Fekete and Szegő then further generalized the estimate $\left|a_{3}-\mu a_{2}^{2}\right|$ with $\mu$ real and $f \in S$. Further, sharp upper bounds for the functional $H_{2}(2)=\left|\begin{array}{ll}a_{2} & a_{3} \\ a_{3} & a_{4}\end{array}\right|=a_{2} a_{4}-a_{3}^{2}$, the Hankel determinant in the case of $q=2$ and $n=2$, known as the second Hankel determinant 
(functional), were obtained for various subclasses of univalent and multivalent analytic functions. Janteng et al. [6] have considered the functional $\left|a_{2} a_{4}-a_{3}^{2}\right|$ and found a sharp upper bound for the function $f$ in the subclass $\mathcal{R}$ of $S$, consisting of functions whose derivative has a positive real part (also called bounded turning functions) studied by MacGregor [9] and have showed that $\left|H_{2}(2)\right| \leq \frac{4}{9}$. For our discussion in this paper, we consider the Hankel determinant in the case of $q=3$ and $n=p$, denoted by $H_{3}(p)$, given by

$$
H_{3}(p)=\left|\begin{array}{ccc}
a_{p} & a_{p+1} & a_{p+2} \\
a_{p+1} & a_{p+2} & a_{p+3} \\
a_{p+2} & a_{p+3} & a_{p+4}
\end{array}\right| .
$$

For $f \in A_{p}, a_{p}=1$, so that, we have

$$
H_{3}(p)=a_{p+2}\left(a_{p+1} a_{p+3}-a_{p+2}^{2}\right)-a_{p+3}\left(a_{p+3}-a_{p+1} a_{p+2}\right)+a_{p+4}\left(a_{p+2}-a_{p+1}^{2}\right)
$$

and by applying the triangle inequality, we obtain

$\left|H_{3}(p)\right| \leq\left|a_{p+2}\right|\left|a_{p+1} a_{p+3}-a_{p+2}^{2}\right|+\left|a_{p+3}\right|\left|a_{p+1} a_{p+2}-a_{p+3}\right|+\left|a_{p+4}\right|\left|a_{p+2}-a_{p+1}^{2}\right|$.

Incidentally, all of the functionals on the right hand side of the inequality (1.4) have known (and sharp) upper bounds except $\left|a_{p+1} a_{p+2}-a_{p+3}\right|$. It was known that if $f \in \mathcal{R}_{p}$, the class of $p$-valent bounded turning functions, then $\left|a_{k}\right| \leq \frac{2 p}{k}$, where $k \in$ $\{p+1, p+2, \ldots\}$ and $\left|a_{p+2}-a_{p+1}^{2}\right| \leq \frac{2 p}{p+2}$, with $p \in \mathbb{N}$.

Motivated by the result obtained by Babalola [1] in finding the sharp upper bound to the Hankel determinant $\left|H_{3}(1)\right|$ for the class $\mathcal{R}$, in this paper we obtain an upper bound to the functional $\left|a_{p+1} a_{p+2}-a_{p+3}\right|$ and hence for $\left|H_{3}(p)\right|$, for the function $f$ given in (1.1), belonging to certain subclass of $p$-valent analytic functions, as follows.

Definition 1 ([13]). A function $f \in A_{p}$ is said to be in the class $I_{p}(\beta)$ ( $\beta$ is real), if it satisfies the condition

$$
\operatorname{Re}\left\{(1-\beta) \frac{f(z)}{z^{p}}+\beta \frac{f^{\prime}(z)}{p z^{p-1}}\right\}>0, \quad z \in E .
$$

(1) Choosing $\beta=1$ and $p=1$, we obtain $I_{1}(1)=\mathcal{R}$.

(2) Selecting $\beta=1$, we get $I_{p}(1)=\mathcal{R}_{p}$.

\section{PRELIMINARY RESUltS}

In this section some preliminary lemmas are stated which are required for proving our results.

Let $\mathcal{P}$ denote the class of functions consisting of $p$, such that

$$
p(z)=1+c_{1} z+c_{2} z^{2}+c_{3} z^{3}+\ldots=1+\sum_{n=1}^{\infty} c_{n} z^{n},
$$

which are analytic in the open unit disc $E$ and satisfy $\operatorname{Re} p(z)>0$ for any $z \in E$. Here $p(z)$ is called Carathéodory function [4]. 
Lemma 1 ([11,12]). If $p \in \mathcal{P}$, then $\left|c_{k}\right| \leq 2$, for each $k \geq 1$ and the inequality is sharp for the function $p(z)=\frac{1+z}{1-z}$.

Lemma 2 ([5]). The power series for $p(z)=1+\sum_{n=1}^{\infty} c_{n} z^{n}$ given in (2.1) converges in the open unit disc $E$ to a function in $\mathcal{P}$ if and only if the Toeplitz determinants

$$
D_{n}=\left|\begin{array}{ccccc}
2 & c_{1} & c_{2} & \cdots & c_{n} \\
c_{-1} & 2 & c_{1} & \cdots & c_{n-1} \\
c_{-2} & c_{-1} & 2 & \cdots & c_{n-2} \\
\vdots & \vdots & \vdots & \vdots & \vdots \\
c_{-n} & c_{-n+1} & c_{-n+2} & \cdots & 2
\end{array}\right|, n=1,2,3 \ldots
$$

and $c_{-k}=\bar{c}_{k}$, are all non-negative. They are strictly positive except for $p(z)=$ $\sum_{k=1}^{m} \rho_{k} p_{0}\left(e^{i t_{k}} z\right)$, with $\sum_{k=1}^{m} \rho_{k}=1$, $t_{k}$ real and $t_{k} \neq t_{j}$, for $k \neq j$, where $p_{0}(z)=\frac{1+z}{1-z}$; in this case $D_{n}>0$ for $n<(m-1)$ and $D_{n} \doteq 0$ for $n \geq m$.

We may assume without restriction that $c_{1} \geq 0$. Using Lemma 2, for $n=2$ and $n=3$, for some complex values $x$ and $z$ with $|x| \leq 1$ and $|z| \leq 1$ respectively, we have

$$
\begin{gathered}
2 c_{2}=c_{1}^{2}+x\left(4-c_{1}^{2}\right) \\
\text { and } 4 c_{3}=c_{1}^{3}+2 c_{1}\left(4-c_{1}^{2}\right) x-c_{1}\left(4-c_{1}^{2}\right) x^{2}+2\left(4-c_{1}^{2}\right)\left(1-|x|^{2}\right) z
\end{gathered}
$$

To obtain our results, we refer to the classical method devised by Libera and Zlotkiewicz [8], which is used by many authors in the literature.

\section{MAin Results}

Theorem 1. If $f \in I_{p}(\beta)(0<\beta \leq 1)$ with $p \in \mathbb{N}$, then

$$
\left|a_{p+1} a_{p+2}-a_{p+3}\right| \leq \frac{2 p}{p+3 \beta} .
$$

Proof. For $f=z^{p}+\sum_{n=p+1}^{\infty} a_{n} z^{n} \in I_{p}(\beta)$, by virtue of Definition 1, there exists an analytic function $p \in \mathcal{P}$ in the open unit disc $E$ with $p(0)=1$ and $\operatorname{Re} p(z)>0$ such that

$$
\begin{aligned}
& (1-\beta) \frac{f(z)}{z^{p}}+\beta \frac{f^{\prime}(z)}{p z^{p-1}}=p(z) \Leftrightarrow(1-\beta) p f(z)+\beta f^{\prime}(z)=p z^{p} p(z) . \\
& (1-\beta) p\left\{z^{p}+\sum_{n=p+1}^{\infty} a_{n} z^{n}\right\}+\beta\left\{p z^{p-1}+\sum_{n=p+1}^{\infty} n a_{n} z^{n-1}\right\}=p z^{p}\left\{1+\sum_{n=1}^{\infty} c_{n} z^{n}\right\} .
\end{aligned}
$$

Upon simplification, we obtain

$$
\begin{array}{r}
(p+\beta) a_{p+1} z^{p+1}+(p+2 \beta) a_{p+2} z^{p+2}+(p+3 \beta) a_{p+3} z^{p+3}+(p+4 \beta) a_{p+4} z^{p+4}+\ldots \\
=p c_{1} z^{p+1}+p c_{2} z^{p+2}+p c_{3} z^{p+3}+p c_{4} z^{p+4}+\ldots
\end{array}
$$


Equating the coefficients of $z^{p+1}, z^{p+2}, z^{p+3}$ and $z^{p+4}$ respectively in 3.2, we have

$$
a_{p+1}=\frac{p c_{1}}{p+\beta} ; a_{p+2}=\frac{p c_{2}}{p+2 \beta} ; a_{p+3}=\frac{p c_{3}}{p+3 \beta} \text { and } a_{p+4}=\frac{p c_{4}}{p+4 \beta} .
$$

Substituting the values of $a_{p+1}, a_{p+2}$ and $a_{p+3}$ from (3.3) in the functional $\left|a_{p+1} a_{p+2}-a_{p+3}\right|$, after simplifying, we get

$\left|a_{p+1} a_{p+2}-a_{p+3}\right|=\frac{p}{(p+\beta)(p+2 \beta)(p+3 \beta)}\left|p(p+3 \beta) c_{1} c_{2}-(p+\beta)(p+2 \beta) c_{3}\right|$.

The above expression is equivalent to

$$
\begin{gathered}
\left|a_{p+1} a_{p+2}-a_{p+3}\right|=\frac{p}{(p+\beta)(p+2 \beta)(p+3 \beta)}\left|d_{1} c_{1} c_{2}+d_{2} c_{3}\right|, \\
\text { where } d_{1}=p(p+3 \beta) ; d_{2}=-(p+\beta)(p+2 \beta) .
\end{gathered}
$$

Substituting the values of $c_{2}$ and $c_{3}$ from (2.2) and (2.3) respectively from Lemma 2 on the right-hand side of (3.4), we have

$$
\begin{aligned}
\left|d_{1} c_{1} c_{2}+d_{2} c_{3}\right|= & \mid d_{1} c_{1} \times \frac{1}{2}\left\{c_{1}^{2}+x\left(4-c_{1}^{2}\right)\right\}+d_{2} \\
& \times \frac{1}{4}\left\{c_{1}^{3}+2 c_{1}\left(4-c_{1}^{2}\right) x-c_{1}\left(4-c_{1}^{2}\right) x^{2}+2\left(4-c_{1}^{2}\right)\left(1-|x|^{2}\right) z\right\} \mid .
\end{aligned}
$$

Using the facts $|z| \leq 1$ and $|x a+y b| \leq|x||a|+|y||b|$, where $x, y, a$ and $b$ are real numbers, which simplifies to

$$
\begin{aligned}
4\left|d_{1} c_{1} c_{2}+d_{2} c_{3}\right| \leq & {\left[\left|\left(2 d_{1}+d_{2}\right)\right|\left|c_{1}\right|^{3}+2\left|d_{2}\right|\left|\left(4-c_{1}^{2}\right)\right|+2\left|\left(d_{1}+d_{2}\right)\right|\left|c_{1}\right|\left|\left(4-c_{1}^{2}\right)\right||x|\right.} \\
& \left.+\left|d_{2}\right|\left|\left(c_{1}+2\right)\right|\left|\left(4-c_{1}^{2}\right)\right||x|^{2}\right] .
\end{aligned}
$$

From (3.5), we can write

$$
2 d_{1}+d_{2}=p^{2}+3 p \beta-2 \beta^{2} ; d_{1}+d_{2}=-2 \beta^{2} .
$$

Substituting the calculated values from (3.7) along with (3.5) on the right-hand side of (3.6), we have

$$
\begin{aligned}
4\left|d_{1} c_{1} c_{2}+d_{2} c_{3}\right| \leq & {\left[\left(p^{2}+3 p \beta-2 \beta^{2}\right) c_{1}^{3}+2(p+\beta)(p+2 \beta)\left(4-c_{1}^{2}\right)\right.} \\
& \left.+4 \beta^{2} c_{1}\left(4-c_{1}^{2}\right)|x|+\left(c_{1}+2\right)(p+\beta)(p+2 \beta)\left(4-c_{1}^{2}\right)|x|^{2}\right] .
\end{aligned}
$$

Since $c_{1}=c \in[0,2]$, noting that $c_{1}-a \leq c_{1}+a$, where $a \geq 0$ and replacing $|x|$ by $\mu$ on the right-hand side of the above inequality, we get

$$
\begin{aligned}
4\left|d_{1} c_{1} c_{2}+d_{2} c_{3}\right| \leq & {\left[\left(p^{2}+3 p \beta-2 \beta^{2}\right) c^{3}+2(p+\beta)(p+2 \beta)\left(4-c^{2}\right)+4 \beta^{2} c\left(4-c^{2}\right) \mu\right.} \\
& \left.+(c-2)(p+\beta)(p+2 \beta)\left(4-c^{2}\right) \mu^{2}\right]=F(c, \mu),
\end{aligned}
$$

for $0 \leq \mu=|x| \leq 1$ and $0 \leq c \leq 2$, where

$$
\begin{aligned}
F(c, \mu)= & \left(p^{2}+3 p \beta-2 \beta^{2}\right) c^{3}+2(p+\beta)(p+2 \beta)\left(4-c^{2}\right)+4 \beta^{2} c\left(4-c^{2}\right) \mu \\
& +(c-2)(p+\beta)(p+2 \beta)\left(4-c^{2}\right) \mu^{2} .
\end{aligned}
$$


Next, we need to find the maximum value of the function $F(c, \mu)$ on the closed region $[0,2] \times[0,1]$. Differentiating $F(c, \mu)$ given in (3.9) partially with respect to $\mu$ and $c$ respectively, we obtain

$$
\frac{\partial F}{\partial \mu}=4 \beta^{2} c\left(4-c^{2}\right)+2(p+\beta)(p+2 \beta)\left(4 c-c^{3}-8+2 c^{2}\right) \mu .
$$

and

$$
\begin{aligned}
\frac{\partial F}{\partial c}= & 3\left(p^{2}+3 p \beta-2 \beta^{2}\right) c^{2}-4 c(p+\beta)(p+2 \beta)+16 \beta^{2} \mu-12 \beta^{2} c^{2} \mu \\
& +(p+\beta)(p+2 \beta)\left(4-3 c^{2}+4 c\right) \mu^{2}
\end{aligned}
$$

For the extreme values of $F(c, \mu)$, consider

$$
\frac{\partial F}{\partial \mu}=0 \quad \text { and } \quad \frac{\partial F}{\partial c}=0 .
$$

In view of (3.12), on solving the equations in (3.10) and (3.11), we obtain the only critical point for the function $F(c, \mu)$ which lies in the closed region $[0,2] \times[0,1]$ is $(0,0)$. At the critical point $(0,0)$, we observe that

$$
\begin{aligned}
\frac{\partial^{2} F}{\partial \mu^{2}} & =-4(p+\beta)(p+2 \beta)<0 ; \\
\frac{\partial^{2} F}{\partial c^{2}} & =-16(p+\beta)(p+2 \beta)<0 ; \\
\frac{\partial^{2} F}{\partial c \partial \mu} & =16 \beta^{2} ; \\
{\left[\left(\frac{\partial^{2} F}{\partial \mu^{2}}\right)\left(\frac{\partial^{2} F}{\partial c^{2}}\right)-\left(\frac{\partial^{2} F}{\partial c \partial \mu}\right)^{2}\right] } & =64\left[(p+\beta)^{2}(p+2 \beta)^{2}-4 \beta^{4}\right]>0,
\end{aligned}
$$

with $p \in \mathbb{N}$ and $0<\beta \leq 1$.

Therefore, the function $F(c, \mu)$ has maximum value at the point $(0,0)$, from (3.9), it is given by

$$
G_{\max }=F(0,0)=8(p+\beta)(p+2 \beta) .
$$

Simplifying the expressions (3.4) and (3.8) together with (3.13), we obtain

$$
\left|a_{p+1} a_{p+2}-a_{p+3}\right| \leq \frac{2 p}{p+3 \beta} .
$$

This completes the proof of our theorem.

Remark 1. Choosing $p=1$ and $\beta=1$ in (3.14), we obtain $\left|a_{2} a_{3}-a_{4}\right| \leq \frac{1}{2}$, this inequality is sharp and coincides with the result of Bansal et al. [2]. 
Theorem 2. If $f \in I_{p}(\beta)(0<\beta \leq 1)$ with $p \in \mathbb{N}$ then

$$
\left|a_{p+2}-a_{p+1}^{2}\right| \leq \frac{2 p}{p+2 \beta}
$$

and the inequality is sharp for the values $c_{1}=c=0, c_{2}=2$ and $x=1$.

Proof. On substituting the values of $a_{p+1}$ and $a_{p+2}$ from (3.3) in the functional $\left|a_{p+2}-a_{p+1}^{2}\right|$, which simplifies to

$$
\left|a_{p+2}-a_{p+1}^{2}\right|=\frac{p}{(p+\beta)^{2}(p+2 \beta)}\left|(p+\beta)^{2} c_{2}-p(p+2 \beta) c_{1}^{2}\right| .
$$

The above expression is equivalent to

$$
\begin{aligned}
& \left|a_{p+2}-a_{p+1}^{2}\right|=\frac{p}{(p+\beta)^{2}(p+2 \beta)}\left|d_{1} c_{2}+d_{2} c_{1}^{2}\right|, \\
& \text { where } \quad d_{1}=(p+\beta)^{2} \quad \text { and } \quad d_{2}=-p(p+2 \beta) .
\end{aligned}
$$

Substituting the value of $c_{2}$ from (2.2) of Lemma 2, applying the triangle inequality on the right-hand side of (3.16), after simplifying, we get

$$
2\left|d_{1} c_{2}+d_{2} c_{1}^{2}\right| \leq\left[\left|\left(d_{1}+2 d_{2}\right)\right|\left|c_{1}\right|^{2}+\left|d_{1}\right|\left|\left(4-c_{1}^{2}\right)\right||x|\right] .
$$

From (3.17), we can write

$$
d_{1}+2 d_{2}=-\left(p^{2}+2 p \beta-\beta^{2}\right) ; d_{1}=(p+\beta)^{2} .
$$

Substituting the calculated values from (3.19), taking $c_{1}=c \in[0,2]$, replacing $|x|$ by $\mu$ on the right-hand side of (3.18), we obtain

$$
\begin{aligned}
& 2\left|d_{1} c_{2}+d_{2} c_{1}^{2}\right| \leq\left[\left(p^{2}+2 p \beta-\beta^{2}\right) c^{2}+(p+\beta)^{2}\left(4-c^{2}\right) \mu\right] \\
& =F(c, \mu), 0 \leq \mu=|x| \leq 1 \text { and } 0 \leq c \leq 2 \text {, } \\
& \text { where } F(c, \mu)=\left(p^{2}+2 p \beta-\beta^{2}\right) c^{2}+(p+\beta)^{2}\left(4-c^{2}\right) \mu \text {. }
\end{aligned}
$$

Now, we maximize the function $F(c, \mu)$ on the closed region $[0,2] \times[0,1]$. Let us suppose that there exists a maximum value for $F(c, \mu)$ at any point in the interior of the closed region $[0,2] \times[0,1]$. Differentiating $F(c, \mu)$ given in (3.21) partially with respect to $\mu$, we obtain

$$
\frac{\partial F}{\partial \mu}=(p+\beta)^{2}\left(4-c^{2}\right)
$$

For $0<\beta \leq 1$, for fixed values of $c$ with $0<c<2$ and $p \in \mathbb{N}$, from (3.22), we observe that $\frac{\partial F}{\partial \mu}>0$. Therefore, $F(c, \mu)$ which is independent of $\mu$ becomes an increasing function of $\mu$ and hence it cannot have a maximum value at any point in the interior of the closed region $[0,2] \times[0,1]$. The maximum value of $F(c, \mu)$ occurs only on the boundary i.e., when $\mu=1$. Therefore, for fixed $c \in[0,2]$, we have

$$
\max _{0 \leq \mu \leq 1} F(c, \mu)=F(c, 1)=G(c) \text {. }
$$


In view of (3.23), replacing $\mu$ by 1 in (3.21), it simplifies to

$$
\begin{gathered}
G(c)=-2 \beta^{2} c^{2}+4(p+\beta)^{2}, \\
G^{\prime}(c)=-4 \beta^{2} c .
\end{gathered}
$$

From the expression (3.25), we observe that $G^{\prime}(c) \leq 0$ for each $c \in[0,2]$ and for every $\beta$ with $0<\beta \leq 1$. Therefore, $G(c)$ becomes a decreasing function of $c$, whose maximum value occurs at $c=0$ only and from (3.24), it is given by

$$
G_{\max }=G(0)=4(p+\beta)^{2} .
$$

Simplifying the expressions (3.16), (3.20) along with (3.26), we obtain

$$
\left|a_{p+2}-a_{p+1}^{2}\right| \leq \frac{2 p}{p+2 \beta} .
$$

This completes the proof of our theorem.

Remark 2. If $p=1$ and $\beta=1$ in (3.27) then $\left|a_{3}-a_{2}^{2}\right| \leq \frac{2}{3}$, this result coincides with that of Babalola [1].

Theorem 3. If $f \in I_{p}(\beta)(0<\beta \leq 1)$ then

$$
\left|a_{p+k}\right| \leq \frac{2 p}{p+k \beta}, \text { for } p, k \in \mathbb{N} .
$$

Proof. Using the fact that $\left|c_{n}\right| \leq 2$, for $n \in \mathbb{N}$, with the help of $c_{2}$ and $c_{3}$ values given in (2.2) and (2.3) respectively, together with the values obtained in (3.3), we get $\left|a_{p+k}\right| \leq \frac{2 p}{p+k \beta}$, with $p, k \in \mathbb{N}$. This completes the proof of our theorem.

Substituting the results of Theorems 1, 2, 3 together with the known inequality $\left|a_{p+1} a_{p+3}-a_{p+2}^{2}\right| \leq\left[\frac{2 p}{p+2 \beta}\right]^{2}$ (see [7]) in the inequality given in (1.4), we obtain the following Corollary.

Corollary 1. If $f \in I_{p}(\beta)(0<\beta \leq 1)$ with $p \in \mathbb{N}$ then

$$
\left|H_{3}(p)\right| \leq 4 p^{2}\left[\frac{2 p}{(p+2 \beta)^{3}}+\frac{1}{(p+3 \beta)^{2}}+\frac{1}{(p+2 \beta)(p+4 \beta)}\right] .
$$

Remark 3. In particular for the values $p=1$ and $\beta=1$ in (3.29), which simplifies to $\left|H_{3}(1)\right| \leq \frac{439}{540}$. This result coincides with that of Bansal et al. [2].

\section{ACKNOWLEDGEMENT}

The author is extremely grateful to the esteemed reviewers for a careful reading of the manuscript and making valuable suggestions leading to a better presentation of the paper. 


\section{REFERENCES}

[1] K. O. Babalola, "On H3(1) Hankel determinant for some classes of univalent functions," Inequal. Theory Appl. (In: Dragomir, S.S., Cho, J.Y. (eds.)), vol. 6, no. 1, pp. 1-7, 2010, doi: $10.1155 / 2008 / 153280$.

[2] D. Bansal, S. Maharana, and J. K. Prajapat, "Third Hankel determinant for certain univalent functions," J. Korean Math. Soc., vol. 52, no. 6, pp. 1139-1148, 2015, doi: 10.4134/JKMS.2015.52.6.1139.

[3] L. D. Branges, "A proof of Bieberbach conjecture," Acta Mathematica, vol. 154, no. 1, pp. 137152, 1985, doi: 10.1007/BF02392821.

[4] P. L. Duren, Univalent Functions. USA: Springer-Verlag New York Inc., 1983, vol. 259, no. 1.

[5] U. Grenander and G. Szegő, Toeplitz Forms and Their Applications; 2nd Edition. New York: AMS Chelsea Publishing Company, 1984.

[6] A. Janteng, S. A. Halim, and M. Darus, "Coefficient inequality for a function whose derivative has a positive real part," J. Inequal. and Appl., vol. 7, no. 2, pp. 1-5, 2006.

[7] D. V. Krishna and T. RamReddy, "Coefficient inequality for certain subclass of p-valent functions," Palestine J. Math., vol. 4, no. 1, pp. 223-228, 2015.

[8] R. J. Libera and E. J. Zlotkiewicz, "Coefficient bounds for the inverse of a function with derivative in P," Proc. Am. Math. Sc., vol. 87, no. 2, pp. 251-257, 1983.

[9] T. H. MacGregor, "Functions whose derivative has a positive real part," Trans. Am. Math. Sc., vol. 104, no. 3, pp. 532-537, 1962, doi: 10.1090/S0002-9947-1962-0140674-7.

[10] C. Pommerenke, "On the coefficients and Hankel determinants of univalent functions," J. Lond. Math. Sc., vol. 41, no. 1, pp. 111-122, 1966, doi: 10.1112/j1ms/s1-41.1.111.

[11] C. Pommerenke, Univalent Functions with a Chapter on Quadratic Differentials by Gerd Jensen. Studia Mathematica/Mathematische Lehrbücher. Band XXV.Vandenhoeck and Ruprecht GmbH and Co KG; 1st edition (December 1974), 1975.

[12] B. Simon, Orthogonal polynomials on the unit circle. Part 1. American Mathematical Society Colloquium Publications, Vol. 54, American Mathematical Society, Providence, RI, 2005. Classical theory: American mathematical society colloquium publications., 2005.

[13] Öznur Ozkan Kilic, "Sufficient conditions for subordination of multivalent functions," J. Inequa. Math. Sc., vol. 2008, no. 3, pp. 1-8, 2008, doi: 10.1155/2008/374356.

\section{Author's address}

D. Vamshee Krishna

Department of Mathematics, Gitam Institute of Science, GITAM University, Visakhapatnam, 530045 , A.P., India

E-mail address: vamsheekrishna1972@gmail.com 